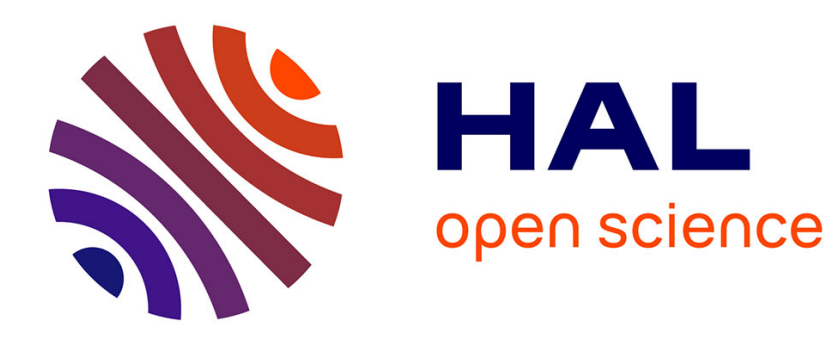

\title{
Jean-Joseph Rabearivelo, poète de l'enracinement et de la nuit
}

Serge Meitinger

\section{To cite this version:}

Serge Meitinger. Jean-Joseph Rabearivelo, poète de l'enracinement et de la nuit. Littérature, 1991, 83, pp.74-88. hal-01413059

\section{HAL Id: hal-01413059 \\ https://hal.univ-reunion.fr/hal-01413059}

Submitted on 9 Dec 2016

HAL is a multi-disciplinary open access archive for the deposit and dissemination of scientific research documents, whether they are published or not. The documents may come from teaching and research institutions in France or abroad, or from public or private research centers.
L'archive ouverte pluridisciplinaire HAL, est destinée au dépôt et à la diffusion de documents scientifiques de niveau recherche, publiés ou non, émanant des établissements d'enseignement et de recherche français ou étrangers, des laboratoires publics ou privés. 


\section{JEAN-JOSEPH RABEARIVELO, POÈTE DE L'ENRACINEMENT ET DE LA NUIT}

\section{MÉTISSAGE ET EXIL INTÉTIEUR}

Comment pouvait-on être Malgache, et qui plus est poète malgache, en cette période des années 1920-1930 où l'Occident toujours conquérant ne doutait pas encore qu'il ne présentât l'unique modèle de civilisation? Jean-Joseph Rabearivelo, à ce jour encore le plus grand poète malgache d'expression française, nous offre à travers sa vie et son œuvre une incarnation exemplaire de ce dilemme qui le déchira tout entier. D’emblée subjugué par la langue et la culture du vainqueur qu'il aima de toute son âme, il partit à l'assaut de la poésie française dont il maîtrisa rapidement les ressorts mais dans le but de plier son nouvel instrument, auquel il imposa bientôt une radicale métamorphose, à l'évocation élégiaque et onirique du territoire natal, de la patrie ou plus exactement de la "matrie" - puisque, pour un Malgache, le pays ne saurait être qu'une terre-mère (firenena).

Jean-Joscph Rabearivelo est né en 1901 à Tananarive - certains disent: en 1903 car il subsiste quelque incertitude sur la date précise, un jugement supplétif obtenu au moment où il cherchait à entrer dans l'administration coloniale permit en effet au poète de se rajeunir. Sa mère était issue de la caste noble (andriana) mérina et apparentée à la plupart des notables de Tananarive mais d'une famille ruinée par l'abolition de l'esclavage en 1896. L'on ne parle guère de son père et l'enfant fut élevé, comme il est de tradition dans ces cas-là, par son oncle maternel, catholique alors que la mère du poète était protestante. Sa scolarité chez les Frères des Ecoles Chrétiennes puis chez les Jésuites du Collège Saint-Michel fut brusquement interrompue alors que l'adolescent n'avait que treize ans et ses études s'arrêtèrent là sans qu'il parût marqué ni par l'enseignement ainsi prodigué ni par le catholicisme de ses éducateurs. Du reste il rejeta presque aussitôt le christianisme et l'essentiel de sa culture fut celle d'un autodidacte. La misère de sa famille le 
contraignit à de nombreux petits métiers et il commença très tôt à écrire en malgache et en français bien qu'en 1921 encore sa maîtrise de la langue française fût toujours incertaine. Il ne trouvera qu'en 1923 ou en 1924 un emploi fixe comme correcteur à l'imprimerie de l'Imerina à Tananarive qu'il conservera jusqu'à sa mort, par suicide, en 1937. Il se marie en 1926, il aura cinq enfants.

Mais ce qui me semble capital dans ce destin de poète c'est que, dans les années 20 de ce siècle, au temps de la Colonie triomphante, un jeune homme de couleur, aristocrate en sa nation bien que ruiné, plein de talent voire de génie, décide de rivaliser avec le colonisateur qui le traite en inférieur, et ce, sur le plan le plus élevé et le plus subtil de la civilisation même : celui de la maîtrise poétique du langage. Prenant au mot le discours colonialiste qui parle abondamment de l'intégration de l'indigène dans le sein de l'Empire, notre jeune poète qui a déjà fait ses preuves dans sa langue maternelle part à la conquête de la langue et de la poésie françaises avec une ardeur d'amoureux. L'évolution de l'autodidacte est prodigieuse : lui qui, en 1921, n'a qu'une pratique chancelante du français, publie dès 1924 un recueil en français, intitulé la Coupe de Cendres, qui dénote chez le poète une pleine capacité à se jouer des mètres et "des rythmes étrangers ». Cette maîtrise ne fera que se confirmer à travers Sylves, paru en 1927, et Volumes, en 1928. Toutefois, celui qui a fait le choix de s'exprimer désormais en français, en ses « années d'apprentissage ", ne remet pas en cause le modèle hérité : il se soumet au chant orné, historié des poètes de la fin du XIX siècle et du début du $\mathrm{XX}^{\mathrm{c}}$. Il retrouve, au gré de son inspiration, l'accent et la cadence du vers romantique, le ton parfois crispé et splénétique de Baudelaire, la vision d'abord plastique des Parnassiens, la tentation de l'excès verbal propre à certains Symbolistes ou la simplicité quelquefois affectée de l'Ecole Romane et de Henri de Régnier. Et, bien que se voulant d'emblée malgache de par ses thèmes, le poète semble chanter souvent sur une lyre d'emprunt dont les échos suscitent le sourire entendu du connaisseur :

Salut, terre royale où mes aïeux reposent, grands tombeaux écroulés sous linjure du temps :

et vous, coteaux fleuris, que des fleuves arrosent

avec leurs ondes d'or aux reflets éclatants!

(Iarive, in Sylves, 1927)

ou

Je suis né près des monts éternels, à lorée du buisson bleu qui mine au village royal, aux rivages d'un fleuve où des ondes moirées bondissent mollement d'un mouvement égal.

(Chants pour l'Amitié, 1931)

La cadence et l'impression, l'harmonieuse mollesse sont toutes lamartiniennes et hormis un audacicux enjambement au début du 
second extrait, qui souligne d'ailleurs le trait ethnique du « buisson bleu » (manga : le bleu est la couleur de la noblesse d'âme et de race et marque le haut rang social), l'on pourrait se croire dans un paysage romantique français pénétré d'aristocratique nostalgie. Mais l'élève est doué, son vers est plein et sonne juste même si « l'air » semble un peu désuet.

Toutefois, même chez Rabearivelo encore débutant, ce choix d'un chant "naissant parmi des rythmes étrangers" n'est pas seulement une option formelle et moins encore l'expression d'un goût exotique - à rebours — , le fond et le message, le sens intime de l'engagement poétique s'y jouent en même temps. "Déporté " hors de sa langue native et se soumettant à une métrique étrangère, à une autre langue qui lui impose son souffle et son héritage littéraire, le poète entre dans l'exil tout autant que s'il avait quitté le sol natal et son chant devenu «étranger " à sa terre ne peut plus évoquer la «matrie " que sur le mode conjoint des Regrets et des Antiquités :

Iarive, Iarive, Iarive la morte, depuis longtemps déja tu m'as fermé la porte destinie à donner sur le soir de ma race!

En vain, parmi le jour qui naît sur la terrasse couronnée autrefois de gerbes corallines, je te cherche an milieu de nos douze collines; en vain, et dans lespoir de retrouver en elles les meilleures de tes grâces simples et belles, je prends part à la ronde ardemment enroulée de tes filles, mes saurs, ô mère désolée: de leur perversion qui me trouble et m'etonne, je ne sais que tresser des fleurs de quel automne en l'bonneur ténébreux de ta mémoire vaine vers qui seules s'en vont ma tristesse et ma peine!

(Iarive, in Volumes, XCI, 1928)

De la sorte, Rabearivelo se fait le Du Bellay des hauts-plateaux malgaches et, l'évocation des " douze collines " royales et sacrées de l'Imerina nous renvoyant aux célèbres sept collines, il pourrait s'écrier - parodiant le Sertorius de Corneille — : « Rome n'est plus dans Rome, elle est toute en mon songe ou en mon poème ". Et le risque, pour le poète engagé sur cette voie, est de ne bientôt plus, s'il persévère, se livrer qu'à une lamentation archéologique et folklorique. Rabearivelo fut conscient de ce danger et l'analyse qu'il fit lui-même poétiquement de son propre malaise lui fit promouvoir l'une des images dominantes de sa quête, celle de l'arbre. En particulier, dans la série de poèmes intitulée Arbres dans Volumes, il compare son sort de poète au cœur déplanté, arraché au terreau originel, à celui d'arbres transplantés d'une région à une autre comme le filao, arbre maritime mal acclimaté aux plateaux de l'Imerina où l'homme l'a contraint de pousser : 
Filao, filao, frère de ma tristesse,

qui nous vient d'un pays lointain et maritime,

le sol imérinien a-t-il pour ta sveliesse

Pélément favorable à ta nature intime?

Maintenant que l'exil fait craquer ton écorce,

P'élan de tes rejets défaillants et sans force

ne dédie aux oiseaux qu'un reposoir sans ombre,

tel mon chant qui serait une auvre folle et vaine

si, né selon un yythme étranger et son nombre,

il ne vivait du sang qui coule dans mes veines!

(Filao, in Volumes, LXXIII)

La problématique de l'identité ethnique, culturelle et linguistique va donc se déployer à travers la métaphore de l'enracinement et du déracinement: mais où, lorsquc l'on est un Malgache des Hautes-Terres, enraciner l'arbre du cœur? Le problème de l'origine se pose à partir de la question du territoire premier, immémorial : d'où viennent les Malgaches? De l'Orient (Indes, Indonésie, Polynésie ?) et de l'Afrique pour leur peuplement primitif, mais aussi de l'Europe pour cette culture qui s'est depuis longtemps infiltrée dans la Grande Ile (dès la fin du XVII' siècle) et qui constitue désormais la seconde nature du poète, lui fournissant les ressources nécessaires à son chant :

$O$ mon caur amoureux de trois zones du monde:

"Europe froide où va le meilleur de toi-même,

IInde au ciel aussi rose et bleu que ton poime,

et $P$ Afrique, to source limpide et profonde,

nul arbre n'a plongé ses vivaces racines

dans le sol différent de trois vergers contraires!

Fixe ton choix sur l une ou lautre de ces terres

bien que toutes, dis-tu, gardent tes origines!

(Volumes, XCII)

Et quel sera alors le territoirc du poème, la patrie où il plongera enfin ses racines s'il veut rester fidèle à l'essentiel ? En est-il d'autre en fait que le champ fertile de la langue natale qui a su, elle, garder les empreintes et les emprunts divers pour les fondre en son creuset mais qui, pourtant, ne répond pas aux critères nouveaux établis par la culture occidentale désormais dominante? Le poète affronte ici la contradiction majeure de toute son entreprise et qui en sera aussi le déchirement majeur. Il a choisi de rivaliser en français avec la culture du vainqueur et il est parvenu à une maîtrise remarquable de l'instrument poétique dont il hérite. Toutefois il est, jusque là jusqu'au début des années 30 - , resté dans la dépendance d'une tradition littéraire qui ne lui permet qu'un rapatriement oblique, sur le mode du thrène et de l'élégie, obéré de plus par une forme inadéquate à toute authentique réappropriation du natal : il n'est encore que le chantre nostalgique d'une dégénérescence qui, par la forme tout autant que par le fond et malgré des sursauts de fierté, reconnaît la défaite d'une race et d'une culture. Il pourrait dcvenir 
le poète officiel de la Colonie, célébrant son pays déchu « selon un rythme étranger et son nombre » et avec la note de passéisme qui convient comme pour mieux rendre hommage à la grande puissance civilisatrice qui, seule, tient les clefs du présent et de l'avenir. Il pourrait aussi décider de revenir en arrière et de s'enfermer dans la langue et la culture de son ethnie, telles qu'elles existent ancestralement. Mais Rabearivelo va s'inventer une voie plus difficile et plus périlleuse - d'une certaine manière il y laissera la vie - : il refuse de sacrifier le français au malgache comme de sacrifier le malgache au français. Cependant, abandonnant résolument le mètre étranger ct le souffle seulement propre à la langue française qui l'anime - le français : " cette langue qui parle à l'âme alors que la nôtre murmure au cœur ", dira-t-il —, le poète se met à écrire ou plutôt à chanter malgacbe en frangais, doublant dc plus toute version française du même poème dans la langue maternelle, elle aussi contrainte à une métamorphose qui n'est pas moindre que celle subie par le français. (Fn fait il est difficile de savoir laquelle des deux versions est première dans le temps réel de la création, mais les deux textes se veulent à chaque fois recto et verso d'une même inspiration essentiellement malgache bien qu'ainsi métissée). De la sorte Rabearivelo tente de concilier en une poésie double et une à la fois, neuve et pourtant très vieille, les exigences de «l'âme » et celles du «cœur », la parole élevée et inspirée et le murmure plus immédiatement sentimental, l'apport français et l'apport malgache, et avec les poèmes bilingues de Presque-Songes, parus en 1934, et ceux de Traduit de la Nuit, parus en 1935, il veut proclamer son retour aux sources: son cour poétique jusque là déplanté et transplanté s'enracine fortement et produit :

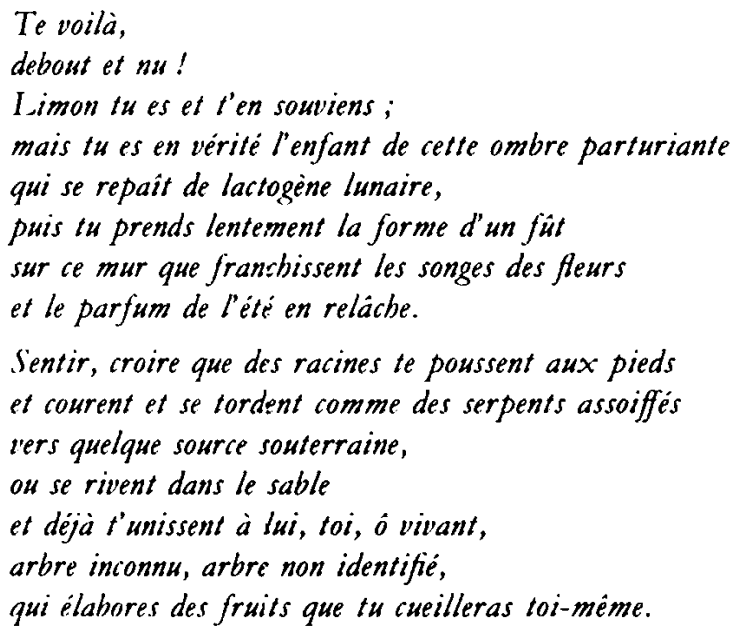

(Traduit de la Nuit, 19 )

Mais de quelle "source souterraine " s'agit-il ici et où s'abreuvent donc ces étranges racines? Quels fruits produit l'homme ainsi interpellé dont la croissance semble se confondre avec celle d'un "arbre inconnu, non identifié "? Il serait trop facile et inexact d'y 
vouloir lire encore, comme dans les premiers recueils, l'essor vigoureux d'un « arbre qui prend racine aux pierres des tombeaux" des ancêtres ( $A$ viarvy in Volumes) car ni le "limon", ni l'" ombre parturiante ", ni la source obscure n'ont ici, pas plus que l'arbre évoqué - ou invoqué - - de nom ni de mémoire et ces racines ne sont sans doute que celles d'un arbre errant qui accompagne les fluctuations de l'ombre nocturne et les mouvements du songe, d'un arbre issu de la nuit, produit dans et par la nuit, et qui vient parfois partager la couche des vivants :

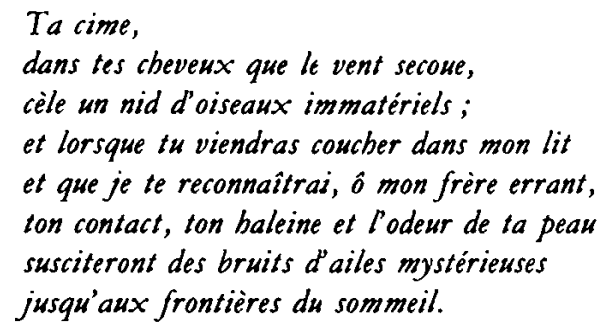

(Traduit de la Nuit, 10)

Car la source mystérieuse - où s'abouchent les vivants et leurs songes, où trempent toutes les racines - est, sans conteste, liée à la nuit des temps et antérieure à toute lumière différenciée, l'immémoriale énigme qui est au principe même de notre univers comme de notre être et que nous pourrions appeler notre arrière-nuit. Ft c'est le nouveau projet de Rabearivelo, métissant la sensibilité malgache et l'instrument poétique français - par osmose et métamorphose réciproques, par le recours à de prodigieuses images et à des rythmes qui font naître des visions et suscitent des monstres plus ou moins mythiques qui ne meurent qu'au matin après une lutte sourde et terrible, images et rythmes "traduits de la nuit " par l'entrelacs des langues et des cultures - que de reconduire ainsi dans et par le lieu du poème la parole poétique jusqu'à l'origine, jusqu'au site premier qui est moins une contrée localisable que l'approche ou du moins l'intuition d'une puissance indéfinie et innommée dont toute la force - inquiétante et bénéfique car elle relève en fait du sacré et de son ambivalence — réside dans son antériorité absolue :

Celle qui naquit avant la lumiere,

est-ce aujourd'bui son septième jour,

aujourd'bui comme bier et comme en l'éternité

sans passé ni futur?

Elle renaît pourtant

avec le sommeil des oiseaux

et tandis que se cachent les pierres blanches

sur les sentiers qu'ont désertés les chèures

comme sur les routes où court le silence.

Mais tu ne vois d'elle que ses myriades d'yeux, ses yeux reptiliens et triangulaires

qui s'ouvrent un à un

entre les lianes célestes.

(Traduit de la Nuit, 21) 
Une source d'être et de lumière - qui est peut-être l'Etre même -, antérieure à la dialectique du jour et de la nuit mais dont le ciel étoilé au dessus de nos têtes nous offrirait les yeux fascinants et vaguement cruels, manifeste sa présence insolite et presque menaçante à chaque tombée de la nuit, faisant ainsi communier notre rythme nycthéméral avec le sans-fond primordial de cette arrière-nuit. Chaque cycle nocturne est ainsi un moment de risque pour la création tout entière - qui ne le sait pas - car la nuit se révèle une dérive sans amarres qui a largué toutes les ancres et mis à nu toutes les racines, une traversée au long cours vers le pur inconnu qui, à chaque fois, ne sauve la possibilité même de l'aube qu'au prix d'une laborieuse gestation : la (re)mise au monde du jour. Et les deux recueils bilingues de Rabearivelo, dont les titres sont en ce sens hautement évocateurs : Presque-Songes et Traduit de la Nuit, instaurent une thématique obsédante du jour et de la nuit, le passage par l'ombre nocturne s'assimilant fréquemment à l'accouchement du jour par l'entremise des songes qui permettent aux oiseaux comme à l'homme de se "nourrir d'astres" donc de s'allier à la lueur persistante de l'arrière-nuit pour vaincre la ténèbre. C'est en effet comme si le retour du soleil, chaque matin, n'allait pas tout à fait de soi, chaque retour du jour étant le résultat d'une traque, d'une quête, le fruit d'une gésine ou d'une couvaison, le " travail " obstiné d'un rêveur cosmique :

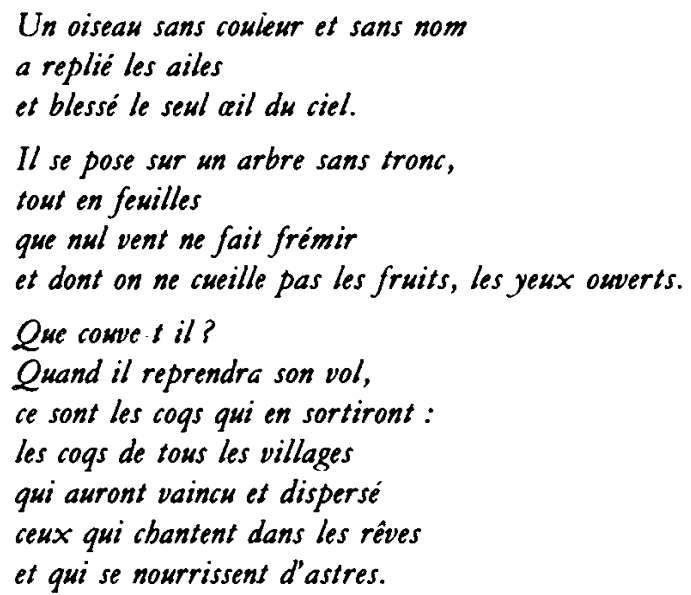

(Traduit de la Nuit, $\sigma$ )

Rabearivelo fait vivre ainsi un extraordinaire bestiaire de la nuit dont l'action et la fonction sont toujours ambivalentes : certaines espèces animales (surtout les oiseaux, "chantres du soleil », et la race entière des bœufs, animaux nobles et honorés dans la tradition malgache) ont pour but de protéger ou de favoriser la naissance du jour, d'autres espèces par contre (pieuvres, araignées...) pactisent avec les ténèbres et participent chaque soir au combat de l'ombre nocturne contre la lumière diurne, mais, tant dans le cas du lever que du coucher du soleil, jamais la victoire d'un élément sur l'autre 
n'est absolue ou définitive : il reste des traces de nuit dans l'éclat du jour et surtout des traces de jour au cœur de la nuit et les animaux effrayants ou répugnants qui ont pris le parti de la ténèbre ne réussissent jamais à annihiler les lueurs stellaires et la prémonition ignée de l'aurore :

Reflux de la lumière océane,

Des poulpes, dans lewr fuite,

noircissent le sable

avec leur bave épaisse;

mais d'innombrables petits poissons

qui ressemblent à des coquillages d'argent,

ne poswant écbapper,

s'y débattent :

ils sont pris dans les rets

tendus par des algues ténébreuses

qui deviennent des lianes

et ervabissent la falaise du ciel.

(Traduit de la Nuit, 7 )

ou encore :

Lente

comme une vache boiteuse

ou comme un taureau puissant

aux quatre jarrets coupés,

une grosse araignée noire sort de la terre

et grimpe sur les murs

puis s'arc-boute péniblement au-dessus des arbres,

Jette des fils qu'emporte le vent,

tisse une toile qui toucbe an ciel,

et tend des rets à travers Pazur.

Où sont les oiseaux multicolores?

Ox̀ sont les chantres du soleil ?

- Les lueurs jaillies de leurs yeux morts de sommeil

dans leurs escarpolettes de lianes,

font reviure leurs songes et leurs résonnances

en cette evanescence de lucioles

qui devient une coborte d'étoiles

pour déjouer l'aracbnéente embâche

que déchireront les cornes $d$ 'un veau bondissant.

(Traduit de la Nuit, 23)

Les métaphores et les métamorphoses liées au cycle nocturne sont aussi variées qu'inattendues mais l'intérêt constamment attaché par le poète à la lueur multiple et une des "étoiles " souligne leur importance symbolique: prises dans des rets d'algues ou de lianes comme dans la toile de l'araignée, perles de verroterie, petits poissons d'argent, torches ou lucioles, elles n'en restent pas moins le regard incarné de l'arrière-nuit primordiale et leur éclat, ravivé par les songes qui s'en nourrissent et les nourrissent, est le pressentiment, l'amorce voire l'adjuvant du matin. Et à l'issue de son ultime recueil Traduit de la Nuit, Rabearivelo se plaît à fonder l'espérance qu'il place en l'avenir sur l'échange, humble mais efficace, réalisé, à la lisière du monde imaginaire propre aux rêves nocturnes, entre les 
hommes vivants et les astres naissants ou sans cesse renaissants :

Mais quel triomphe certain

m'annoncent deja tous ces signaux

que terre et ciel s'envoient

à l'orée du sommeil :

dans nos cités de vivants

jusqu' aux plus bumbles buttes

répondent aux appels de feu

jaillis des étoiles naissantes.

(Traduit de la Nuit, 30)

Toutefois une telle harmonie, qui conjugue pacifiquement la force pure et haute d'un onirisme actif avec la force principielle du cosmos, n'est pas toujours si facile à instaurer. Le jeune « veau bondissant ", nommé juste à la fin du poème mettant en scène la " grosse araignée noire » et qui désigne le matin, représente certes le sursaut natif du jour, violemment espéré, et la puissance vitale propre à la lumière s'arrachant à l'ombre, matricielle mais aussi mortifère ; néanmoins, malgré l'aide des rêves alliés aux astres de la nuit, il faut aussi parfois accomplir un sacrifice en règle pour produire le miracle quotidien de l'aube :

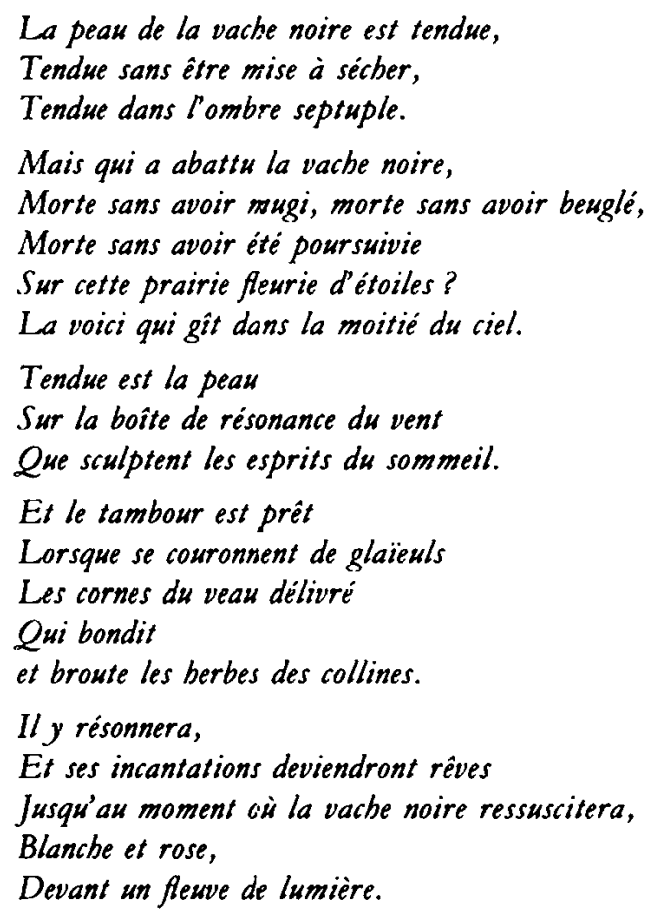

(Traduit de la Nuit, 3)

Ce poème, l'un des plus célèbres et des plus beaux de Traduit de la Nuit, est très clairement une métaphore de la nuit présentée comme le passage nécessaire par une mort symbolique et sacrificielle et donnée, en même temps, comme le lieu privilégié d'un rituel de résurrection destiné à permettre la naissance du jour - qui est comme une "naissance de dieu sans temple ", selon les termes d'un poème ancien de la Coupe de Cendres (Matin). L'animal mystérieusement sacrifié devient ici un être céleste ou plutôt un médiateur 
entre le ciel étoilé (qu'il se met alors à représenter) et l'humain : sa peau tendue sur la voûte du ciel se métamorphose en un tambour cosmique dont le battement rituel - mi-songe, mi-réalité accompagnera et facilitera le bond natal du "veau déliv ré " qui n'est qu'un avatar, chaque jour nouveau, chaque jour premier, de la vache chaque soir immolée. Et tout, ici, s'accomplit selon le rite le plus strict et le plus mesuré : la noblesse naturelle de la « vache-mère du bouf " (reniomby) se plie avec simplicité et abnégation à la rigueur d'un supplice qu'elle accepte dans l'intérêt de l'élan vital qui la transcende et la rédime. Cependant le sacrifice nécessaire ne revêt pas toujours cette ordonnance sans faille et la victime déchirée peut aussi donner l'impression de s'être vainement débattue contre une souffrancc imposée dont elle ne paraît pas comprendre la portée :

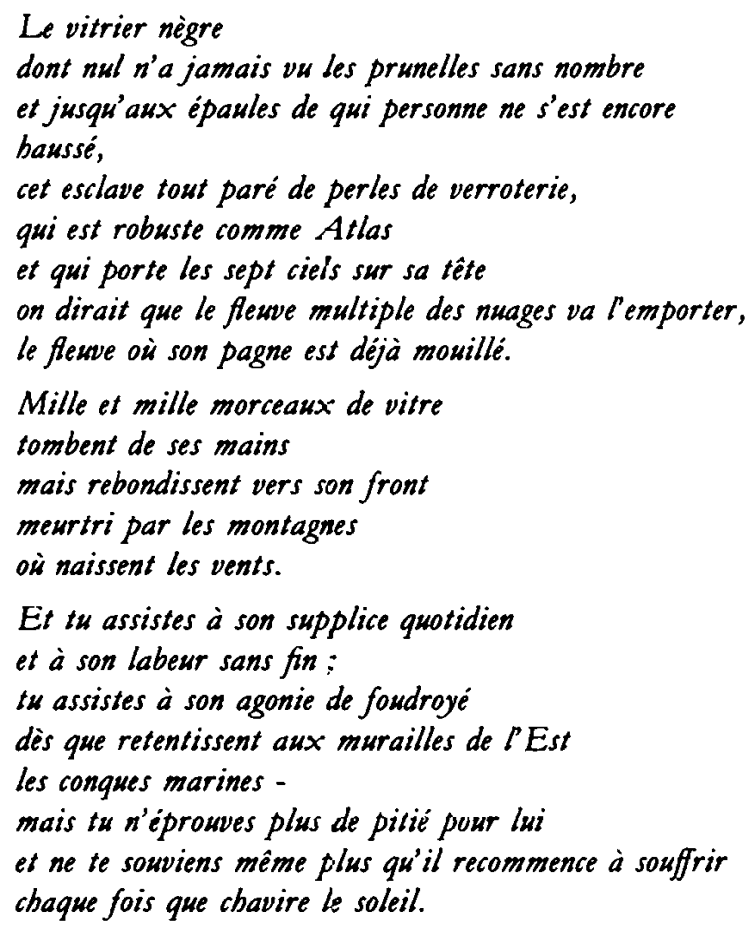

De fait l'évocation de l'esclave, vitrier cosmique victime de sa fonction, insiste plus en ce cas sur le supplice et l'agonie de l'être sacrifié dont les souffrances sont décrites que sur la résurrection radieuse du jour. Ici encore le géant évoqué, porteur des sept ciels et comparé à Atlas, est une image du ciel nocturne mais la perversité des étoiles dont il soutient la voûte est mise en évidence : leurs éclats contribuent aussi à le blesser et à le déchirer. Un fois encore cette agonie est la parturition d'un jour nouveau, mais le témoin de cette souffrance céleste où la nuit est comme "en travail » du jour (qui proprement « l'achève ») veut rester insoucieux et indifférent devant cette monstrueuse gésine : il oublie avec quelque mauvaise conscience le «labeur » douloureux et méprisé de celui qui se débat seul dans la ténèbre, il oublie la souffrance du négatif comme si 
momentanément la portée et la nécessité de ce « supplice quotidien » lui échappaient, à lui aussi. Toutefois cette indifférence, affichée par le poète envers les terribles conditions propres à chaque éclosion matinale, est sans doute liée au souhait plus ou moins conscient qu'il fait d'oublier la portée et la nature d'un supplice qui est aussi le sien et je voudrais lire dans cette dénégation - qui ne masque en rien la gravité de ce qu'elle tente de nier - un aveu à rebours : pris entre la promesse des étoiles et leur secrète menace, entre l'arrière-nuit entrevue, la nuit peuplée de monstres ou de prodiges et la mise au monde du matin, le poète se sent voué à un «travail » analogue à celui du vitrier nègre, à un péril sans cessc croissant. Et il faut sans doute prendre au pied de la lettre la teneur de l'exergue, emprunté à Jules Supervielle et placé à l'orée de Traduit de la Nuit :

Pour avoir mis le pied

Sur le cour de la nuit

Je suis un bomme pris

Dans les rets étoiles.

En effet qu'en est-il du grand pari de Rabearivelo associant rapatriement et métissage à travers l'expérience de ses deux derniers recueils? Le retour aux sources ethniques est évident de par les paysages, les personnages, les animaux, les plantes, les choses, les coutumes et les rites caractérisés de l'intérieur par le trait le plus fin et le plus exact. L'atmosphère de la plupart des textes, même de ceux qui sont le plus fortement imprégnés de lyrisme onirique, reste malgache. Mais, bien que jouant systématiquement du «miroitement des images " et de la polysémie, il ne s'agit pas ici de poèmes vraiment analogues aux bainteny de la tradition mérina. Car si le bainteny fait bien, lui aussi, tenir dans le minimum de matière verbale le maximum de sens et d'allusions possibles, héritier ethnique d'une "scicnce des mots ", il reste incompréhensible s'il n'est pas soigneusement "situé » et éclairé par les circonstances mêmes de son énonciation dans l'histoire et la culture de l'ethnie comme dans celles des hommes à qui s'adresse concrètement le poème : face au bainteny, c'est à chacun d'exercer en situation sa sagacité et sa vivacité intellectuelle, ces dernières seront avant tout, avant même le plaisir pris à la beauté verbale, un gage d'intégration dans la communauté traditionnelle. Or les images promues par Rabearivelo ne s'épuisent ni ne se fixent jamais par la référence culturelle ou ethnique, une et multiple, qu'on peut leur trouver; elles ouvrent au contraire par leurs entrelacs en réseaux un monde nouveau qui a, par rapport aux mythes, aux rites et aux réalités malgaches évoqués, son autonomie et sa personnalité propre parce qu'il les englobe tout en les dépassant, désignant de la sorte une origine encore plus originelle que le propre ethnique. Une preuve a contrario de l'originalité du poète en la matière se trouve dans ses Vieilles Chansons des pays d'Imerina 
(parues en 1939, après la mort du poète) : traduisant en français un certain nombre de bainteny traditionnels, Rabearivelo qui vise plus à une traduction littéraire, universellement compréhensible, qu'à l'exactitude ethnographique n'arrive pas le plus souvent - malgré un évident effort en ce sens - à donner aux courts textes qu'il rend ainsi en français l'unité de ton, la cohérence syntaxique et métaphorique, la perspective globale qui font de chaque poème un tout à part entière, qualités qui sont celles de ses propres œuvres. Dans la perspective malgache toujours, le modèle métrique, lui non plus, ne correspond pas aux moules hérités des anciens : il ne s'agit en effet ni de vers mesurés et rimés (à la mode européenne) ni de vers fondés sur le schéma accentuel des récitations ancestrales. Enfin, souvent le poc̀tc parle français en malgache parce qu'il emploie volontiers des termes abstraits, désignant des notions ou des sentiments - termes souvent recomposés de manière savante à partir des racines primitives (sur un modèle privilégié par l'Académie Malgache pour faire évoluer et enrichir la langue).

De même sur l'autre versant, toutes les conventions du vers français se trouvent débordées: les vers libres, ici à l'œuvre, inventent leur rythme au fur et à mesure de leur progrès et, bien que la découpe rythmique se superpose le plus souvent à la découpe syntaxique, un certain nombre de disjonctions et d'enjambements s'ingénient, à point nommé, à disloquer tous les enchaînements par trop convenus et à dérouter tout automatisme. De plus, souvent le poète parle malgache en français: des expressions ou des mots composés malgaches littéralement traduits font image tout en gardant leur sens propre malgache, car, si le voisinage du français incite le malgache à inventer des termes et des modes d'expression abstraits parfois arides pour le non-initié, inversement le malgache remet le français sur la voie d'une langue plus sensuelle et plus concrète. Enfin il ne suffit pas d'invoquer une hypothétique influence surréaliste pour justifier le déferlement des images car, chez Rabearivelo, les réseaux métaphoriques conservent une forte unité sémantique et s'interdisent toute association gratuite au point de constituer clairement et distinctement de petits systèmes planétaires avec, chacun, leurs lois de gravitation interne. On le voit, le retour aux sources s'accompagne d'une métamorphose radicale des dites sources et d'un dépassement inédit de toutes les limitations préexistantes et c'est en ce point même que croît le danger. Un tel rapatriement qui débordait visiblement le seul retour à la " matrie " pour aller plus loin encore vers l'originel, un tel métissage qui retravaillait les deux langues et les deux cultures pour leur faire dire et porter ce que, séparément et même ensemble, elles n'avaient encore jamais su dire, ne risquaient-ils pas de passer pour une double trabison? 
Et en fait ce fut ce qu'il se passa. Les autorités coloniales ne firent aucun effort pour mettre leurs actes en accord avec le discours officiel sur l'intégration des indigènes : elles refusèrent obstinément au poète le modeste poste administratif qu'il sollicitait, il ne put non plus faire partie de la délégation malgache à l'Exposition Universelle de 1937. L'autodidacte avait sans doute par trop outrepassé le modèle scolaire en vigueur: l'on douta en haut lieu de son conformisme idéologique et de son obéissance. Mais du côté de ses compatriotes l'accueil ne fut pas plus ouvert : la bourgeoisie mérina, dont il était socialement proche par la naissance mais qu'en tant que poète et que romancier il ne ménagea jamais, tout à son désir servile d'imiter le vainqueur, était pour le moins gênée par sa revendication sans fard de malgachité tout en jalousant sa maîtrise de la langue et de la pensée françaises; par contre l'intelligentsia nationaliste lui faisait le reproche d'être, jusque dans la lettre et l'idée de ses derniers poèmes en malgache - ceux de Presque-Songes et de Traduit de la Nuit - trop imprégné encore d'esprit français et de cantonner son engagement patriotique au seul plan de l'imaginaire. Et tous ces faits qui ont entraîné chez le poète le sentiment d'être incompris sur l'essentiel, l'isolement moral et social qui en résulta (malgré quelques belles amitiés) ainsi que la misère et son cortège d'humiliations, une vie agitée de pulsions violentes et conduite sans aucun ménagement, le sentiment toujours présent de la mort depuis le décès de sa fille Voahangy en 1933 sont autant de causes plausibles du suicide du 22 juin 1937, qui, comme tout suicide, reste un entier mystère.

Je me demande toutefois si, à toutes ces causes vraisemblables, il ne faudrait pas en ajouter une autre qui tiendrait plus spécifiquement à la nature et à la portée de l'engagement poétique lui-même tel que nous l'avons décrit. Car nous avons vu comment, ayant pris conscience, dans et par l'expérience même du poème, de la présence presque indistincte d'une arrière-nuit au sein même de la nuit antériorité absolue, incréée, seule garante de la (re)naissance du jour et de la vie - Rabearivelo lui a fait formellement allégeance et comment il s'en est de la sorte remis à l'ambivalence active qu'une telle puissance déploie à l'orée du songe, sous l'invocation certes de l'imaginaire mais avec néanmoins une influence réelle sur l'« âme " et sur le "cœur" du rêveur produisant le monde inouï de son rêve. Et les prestiges de la "nuit" comme ceux de l'arrière-nuit, ici traduits, l'affirmation sacrée du firmament, la couleur des songes ct la promesse de l'aube se sont donnés, avant même que de se faire les métaphores d'une prodigieuse gésine, pour les signes universels d'une présence au monde qui est fraîcheur et saisie de l'être à l'état naissant, sensible à l'«âme " et au "cœur " de celui, quel qu'il soit, qui en partage la révélation. Mais alors ce n'est plus seulement - 
comme dans le bainteny - l'ethnie parlant à travers les paroles consacrées du poète qui explique l'ethnie à elle-même, plus seulement le métis ou le lettré bilingue qui conjugue les langues pour le plaisir d'autres lettrés, mais le sujet individuel, infiniment exposé, qui traduit et trabit les mots, les rites, les symboles de l'ethnie, qui détourne les traits les plus typiques des langues et des cultures aimées pour en faire un usage à la fois intime et universel. Et, de la sorte, le poète, usant de sa libre subjectivité créatrice, ne fait, à nos yeux, qu'ajouter une variante - ou une variation - personnelle et universelle aux deux traditions dont il est nourri. Cependant, et c'est là, nous semble-t-il, le drame propre à Rabearivelo, il n'est pas vraiment sûr qu'une telle entreprise soit au regard de ses ancêtres légitime: il vit sa tentative - sa tentation - trop subjective et trop universaliste comme un déchirement, un point d'angoisse et de déréliction, comme la cause profonde et le lieu-même de la punition infligée par l'esprit de ses morts à l'enfant qui les trahit.

Car le rite sacrificiel, le supplice pensé comme la gestation infinie d'une aurore encore insue ont sans doute aussi pour dessein comme nous le soulignions plus haut à propos du "vitrier nègre" - de nous dire quelque chose du grand péril que le poète eut constamment le sentiment d'affronter : de dire le déchirement d'un créateur qui - s'éprouvant à la fois malgache et autre que malgache, métis et plus que métis, mais non encore réconcilié, réunifié - voudrait sortir de la parturition infinie et douloureuse de son être pour atteindre aux rives de l'aube, de son aube. Comme le géant cosmique, il lui semble s'être débattu entre plusieurs patries ou " matries ", plusieurs langues et cultures et il ne veut plus rien savoir de ce tourment qui l'excède car l'accouchement de l'aurore ne se fait pas ici au bénéfice de la victime consacrée. Mais, comme la "vache noire" cette fois, il put aussi concevoir l'holocauste, son bolocauste volontaire de juin 1937, comme un rite destiné à replacer la victime dans le sein de sa race qu'elle honore ainsi par sa soumission au sacrifice, par la noblesse simple et mesurée de son attitude conforme aux règles ancestrales. Et dans ce cas la victime ressuscite : elle réintègre le monde de la lumière et de la vie.

Nous ne savons pas bien-sûr de quelle nature était l'espoir ou le désespoir du poète au moment où il ordonna si minutieusement le rituel de sa propre mise à mort, mais nous savons par ailleurs quel symbole fut toujours pour lui celui de la lumière stellaire associée à l'aurore. Or, dans un poème de Presque-Songes dédié à la gloire de l'aube, il érige une fois encore le matin en emblème et en fait le signe même et le tombeau de l'artiste: redevenu indivis, l'éclat de la lumière incréée - gemme absolue que la nuit s'efforce encore mais en vain de tailler et de détailler - retrouve dans et par l'éclosion du jour la plénitude de son être et de l'être mais c'est au prix sans doute 
de la mort du poète :

Fondues ensemble toutes les étoiles

dans le creuset du temps,

puis refroidies dans la mer

et sont devenues un bloc de pierres a facettes.

Lapidaire moribonde, la nuit,

$y$ mettant tout son caur

et tout le regret qu'elle a de ses meules

qui se désagrègent, se désagrègent

comme cendres au contact du vent

taille amoureusement le prisme.

Mais c'est une stèle lumineuse

que l'artiste a érigé sur sa tombe invisible.

(Une autre [naissance du jour])

\section{NOTE BIBLIOGR APHIQUE}

Grâce à la petite collection "Orphée " publiée aux éditions de La Différence (Paris) et grâce à la collection « Monde noir " aux éditions Hatier (Paris), Rabearivelo est de nouveau lisible en Europe et dans toute la francophonie : Traduit de la nuif et autres poimes, $1990, \mathrm{n}^{\circ} 39$ de la collection "Orphée " et Presque-Songes, Traduit de la nuit, Chants pour Abéone, édition bilingue, Hatier, 1990.

A la fin de 1989, ont paru à Marseille, aux éditions Sud les Actes du Colloque Jean-Joseph Rabearivelo, cet inconnu? qui avait eu lieu à Tananarive en mai 1987. 